See discussions, stats, and author profiles for this publication at: https://www.researchgate.net/publication/335961722

\title{
Modelling incomplete fusion dynamics of complex nuclei at Coulomb energies
}

Article in Physical Review C · October 2019

DOI: 10.1103/PhysRevC.100.044604

\section{CITATIONS}

0

2 authors:

Rafael Van den Bossche

University of Surrey

3 PUBLICATIONS 38 CITATIONS

SEE PROFILE

Some of the authors of this publication are also working on these related projects:
READS

135

Alexis Diaz-Torres

University of Surrey

126 PUBLICATIONS 2,042 CITATIONS

SEE PROFILE

Project $\quad$ Breakup of light weakly bound nuclei View project

Project Modelling incomplete fusion of complex nuclei at Coulomb energies: Superheavy element formation View project 


\title{
Modelling incomplete fusion dynamics of complex nuclei at Coulomb energies
}

\author{
Rafael Van den Bossche $\odot$ and Alexis Diaz-Torres $\odot$ \\ Department of Physics, University of Surrey, Guildford GU2 7XH, United Kingdom
}

(Received 4 August 2019; published 4 October 2019)

\begin{abstract}
The incomplete fusion dynamics of ${ }_{10}^{20} \mathrm{Ne}+{ }_{82}^{208} \mathrm{~Pb}$ collisions at energies above the Coulomb barrier are investigated using a novel semiclassical dynamical model, which combines a classical trajectory model with stochastic breakup, as implemented in the PLATYPUS code, with a dynamical fragmentation theory treatment of two-body clusterization and decay of a projectile. A finite-difference method solution to the time-independent Schrödinger equation in the charge asymmetry coordinate is employed by way of diagonalizing a tridiagonal Hamiltonian matrix with periodic boundary conditions. Results are compared with published experimental values to indicate the success of this new model, and next steps for its development are detailed.
\end{abstract}

DOI: 10.1103/PhysRevC.100.044604

\section{INTRODUCTION}

Superheavy elements (SHEs) often refers to the transactinide elements, which have an atomic number $104 \leqslant Z \leqslant$ 120 , and sometimes to the superactinide elements $(121 \leqslant$ $Z \leqslant 157)$ and beyond [1]. In some cases the term has been used to refer to elements located in or near the theoretically predicted island of stability, which have atomic and mass numbers $(Z, N) \cong(114$ or 120 or 126,184$)$ [2,3], and in 1990 Seaborg and Loveland suggested that the term SHE should be associated with "an element whose lifetime is strikingly longer than its neighbors in the chart of the nuclides" [4]. The aforementioned island of stability is a predicted set of heavy nuclides with a near magic number of protons and neutrons that temporarily reverses the trend of decreasing stability (with increasing atomic and neutron numbers) in elements heavier than uranium [5]. In the context of this work, the term SHEs refers to the transactinide elements, which includes elements located in or near the island of stability.

SHEs were predicted using the nuclear shell model in the 1960s [2,6,7], and their production is very challenging (due to very small cross sections in the range of a few picobarns or less), with complete fusion (CF) of heavy ions being one of the most successful ways of producing SHEs [8]. The CF mechanism predominantly produces neutron-deficient SHEs, making investigation into new methods of production crucial for further progress in SHE research.

The incomplete fusion (ICF) mechanism differs from the $\mathrm{CF}$ mechanism in that the projectile undergoes breakup and at least one, but not all, of the fragments fuse with the target as opposed to the projectile wholly fusing with the target, with or without undergoing breakup. A complex nucleus is a nucleus wherein it is not clear that there is one dominant cluster structure, as opposed to a weakly bound light nucleus (such as ${ }^{6} \mathrm{Li},{ }^{7} \mathrm{Li}$, and ${ }^{9} \mathrm{Be}$ ), which has a single dominant cluster structure. A complex nucleus can be viewed as a superposition of many simple cluster structures, and this is why fragmentation theory [9-11] is useful for this work.
This work is experimentally motivated: the observation of energetic $\alpha$ particles at forward angles in reactions induced by heavy ions at Coulomb energies [12-15] indicates the existence of a reaction mechanism in which, following projectile breakup, the $\alpha$ particle carries away most of the bombarding energy of the projectile, leaving the other remaining projectile fragment to be captured by the target resulting in a colder fusion product than would typically be achieved via the CF mechanism (with higher excitation energy). The low excitation energy of these cold products from ICF reactions results in both a higher survivability against fission and fewer neutrons evaporated, indicating that this mechanism could be a successful way of producing relatively stable SHE isotopes.

In order to understand the underlying dynamics, numerous dynamical models were proposed following the first experimental observation of projectilelike fragments associated with ICF [16,17]. A breakup fusion model [18] based on the distorted-wave Born approximation was proposed by Udagawa and Tamura wherein the projectile is assumed to break up into $\alpha$ clusters within the nuclear field of the target, one of which fuses with target nucleus. The production of these breakup fragments was described by a simple planewave-projectile-breakup model $[19,20]$ proposed by $\mathrm{Wu}$ and Lee wherein the (fast) breakup process is governed by the projectile's nucleon momenta distribution, and the coupling of Fermi momentum and the center-of-mass momentum is assumed to result in the production of these quick fragments. A sum-rule model [21] proposed by Wilczyński et al. that concluded that ICF mainly originates from peripheral collisions and is confined to the $l$ space above the $l_{\text {crit }}$ for $\mathrm{CF}$ was later extended by Brâncuş et al. [22]. Bondorf et al. proposed a promptly-emitted-particles model [23] in which it was explained that the nucleons transferred to the target nucleus from the projectile nucleus may obtain extra velocity to escape before equilibration as a consequence of being accelerated in the nuclear field of the target. Fermi-jet [24,25], moving-source [26], exciton [27,28], and overlap [29,30] models, as well as dynamical models for ICF and projectile breakup [31,32], were also proposed. The probability of 
ICF was correlated with the mass asymmetry of interacting partners by Morgenstern et al. [33], a supplement for which was presented by Gupta et al. [34] and Singh et al. [35]. The particle- $\gamma$ coincidence measurements by Inamura et al. [36-38] and Zolnowski et al. [39] resulted in the advancement of understanding of ICF dynamics. Geoffroy et al. investigated the origin of projectile fragments from undamped peripheral interactions at high $l$ values, and measured the $\gamma$ multiplicity as well as the correlation of energies and angles of charged particles [40]. Trautmann et al. [41] and Inamura et al. [36-38] also emphasized the peripheral nature of ICF. It was inferred in Gerschel's review of ICF [42] that target deformation also has an effect on localization of the $l$ window. The emission of projectilelike fragments was suggested to originate from $l$ values smaller than $0.5 l_{\text {crit }}[43,44]$ based on results with semimagic targets obtained by Tricoire et al. [25], however, this emission was found to originate from high $l$ values for rare-earth targets $[21,36,40,45]$. Despite the aforementioned studies, ICF dynamics are still not very well understood at energies around 4-7 MeV/A [46,47].

As this mechanism has not been thoroughly explored yet, and could prove to be an effective way of producing neutronrich SHE isotopes with low excitation energies [14], the focus of this work is to investigate ICF reaction dynamics of complex nuclei at Coulomb energies. Broadly, this reaction mechanism can also be useful for producing new isotopes throughout the periodic table [48].

To this aim, a semiclassical dynamical model is being developed by combining a classical trajectory model with stochastic breakup, as implemented in the PLATYPUS code [49], with the quantum-mechanical fragmentation theory [50] treatment of two-body clusterization and decay of a projectile. A finite-difference method solution to the time-independent Schrödinger equation in the charge asymmetry coordinate is being explored by way of diagonalizing a tridiagonal Hamiltonian matrix with periodic boundary conditions.

\section{BACKGROUND AND THEORY}

\section{A. Background}

PLATYPUS is a self-contained FORTRAN-90 program based on a classical trajectory model $[49,51]$ with stochastic breakup and is a powerful tool for quantifying complete and incomplete fusion, as well as breakup in reactions induced by weakly bound two-body projectiles near the Coulomb barrier, which is to be extended for the reactions of complex nuclei.

The program calculates a wide range of observables including integrated $\mathrm{CF}$ and ICF cross sections and their spin distribution, as well as breakup observables such as the angle, kinetic energy, and relative energy distributions of the fragments. All of the observables are calculated using a threedimensional classical dynamical model merged with Monte Carlo sampled probability-density distributions [49].

A crucial input of the model implemented in the PLATYPUS code is an experimentally determined breakup function, which encodes the effects of the Coulomb and nuclear interactions that cause the projectile breakup. For example, a ${ }^{9} \mathrm{Be}$ projectile (made up of two $\alpha$ particles and a neutron) breaks up upon striking a stable target, and the resultant-particles are detected

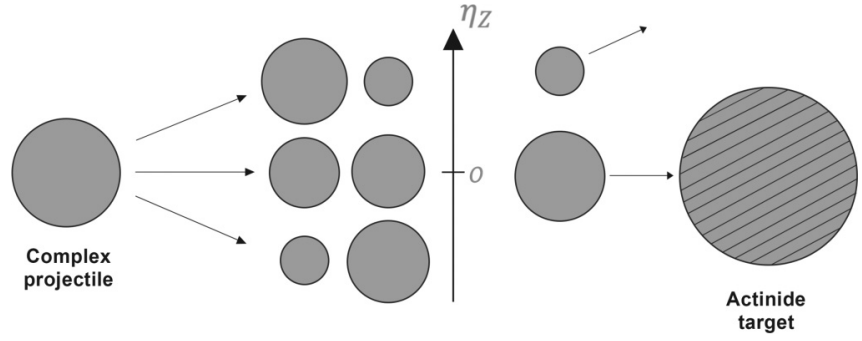

FIG. 1. A diagram representing ICF of a complex projectile (undergoing fragmentation in the charge asymmetry coordinate) and an inert target.

for a given scattering angle of the projectile. The probability of breakup is defined as a function of the distance of closest approach $R_{\min }$, by dividing the experimental breakup cross section by the Rutherford scattering cross section [52]. The integration of a local probability density over the projectiletarget distances results in this total breakup probability [49]:

$$
P_{B U}\left(R_{\min }\right)=2 \int_{R_{\min }}^{\infty} \mathcal{P}_{B U}^{L}(R) d R=e^{-\alpha R_{\min }+\beta},
$$

where $P_{B U}$ is the probability of breakup, $\mathcal{P}_{B U}^{L}(R) d R$ is the probability of breakup in the interval $R$ to $R+d R$, and $\alpha$ and $\beta$ are constants determined from experimental results that vary for different systems $[53,54]$. This function is sampled to determine the position of breakup in the orbit of the projectile [49].

A major limitation of using the PLATYPUS code to model the ICF of complex nuclei is that it requires the binary fragmentation configuration of the projectile as an input; in effect it addresses the ICF of a single binary fragmentation configuration. While the code provides the probability of a given projectile breaking up into a specific binary configuration of fragments (as a function of $R_{\min }$ ), it does not mean that the projectile would necessarily break up into those two specific fragments in reality as many other competing binary fragmentation configurations are possible with a complex nucleus (see Fig. 1). This shortcoming is the motivation for adopting a dynamical fragmentation theory [50] treatment of two-body clusterization and decay of a projectile.

\section{B. Charge asymmetry}

In 2012 Kuklin et al. presented a model [50] that makes use of the charge asymmetry coordinate, $\eta_{Z}$, which stems from the mass asymmetry coordinate, $\eta$, first proposed by the Frankfurt School for Theoretical Nuclear Physics in the 1970s $[10,11]$, and is equal to the continuous volume asymmetry coordinate when the two nuclei overlap $[10,11]$. Take, for example, a complex projectile undergoing fragmentation in order to incompletely fuse with an actinide target, as shown in Fig. 1.

In the center of Fig. 1 there are three binary configurations shown for the fragmentation of the projectile in the charge asymmetry coordinate, to demonstrate the concept. $\eta_{Z}$ is defined as the difference in the charges of the two fragments 


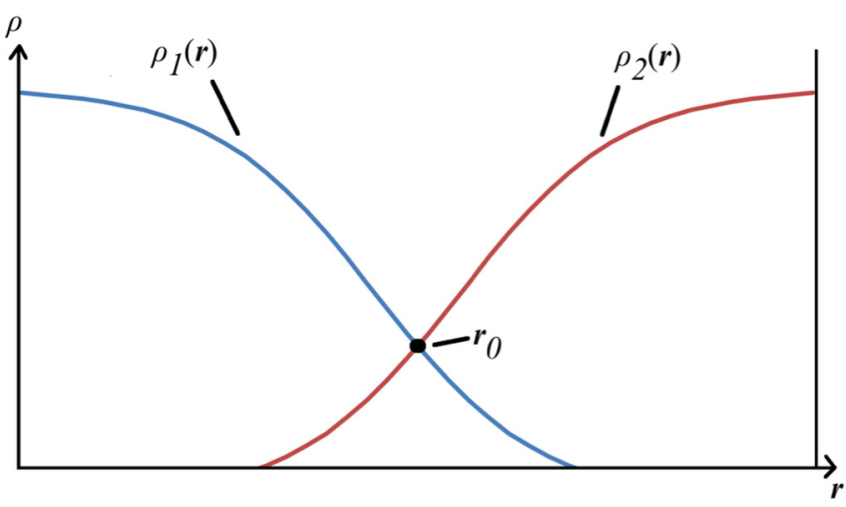

FIG. 2. Schematic diagram representing the overlap between intranuclear nucleon-distribution tails of the two fragments (the neck) of a dinuclear system.

divided by the sum total of their charges, as per Eq. (2):

$$
\eta_{Z}=\frac{\left(Z_{1}-Z_{2}\right)}{\left(Z_{1}+Z_{2}\right)}
$$

where $Z_{1}$ and $Z_{2}$ are the charges of fragments 1 and 2, respectively. In the case of $\eta_{Z}=0$, the fragmentation is symmetric $\left(Z_{1}=Z_{2}\right)$, as per the middle configuration in Fig. 2. At the extremes, where $\eta_{Z}= \pm 1$, there is no fragmentation (as one of the would-be fragment has charge $Z=0$ ). The charge asymmetry coordinate is similar to the mass asymmetry coordinate [9-11], but it concerns charge distribution rather than mass distribution:

$$
\eta=\frac{\left(A_{1}-A_{2}\right)}{\left(A_{1}+A_{2}\right)} .
$$

The determination of the state of a dinuclear system for a given parent nucleus is made by solving the time-independent Schrödinger equation in the charge asymmetry coordinate with periodic boundary conditions at $\eta_{Z}= \pm 1$ [50]:

$$
\hat{H} \Psi_{n}\left(\eta_{Z}\right)=E_{n} \Psi_{n}\left(\eta_{Z}\right)
$$

where $\Psi$ is the wave function, $E$ is the energy, $n$ is the eigenstate quantum number, $\eta_{Z}$ is the charge asymmetry coordinate, and $\hat{H}$ is the collective Hamiltonian:

$$
\hat{H}=-\frac{\hbar^{2}}{2} \frac{\partial}{\partial \eta_{Z}}\left(B^{-1}\right)_{\eta_{Z} \eta_{Z}} \frac{\partial}{\partial \eta_{Z}}+V\left(\eta_{Z}\right)
$$

where $\left(B^{-1}\right)_{\eta_{Z} \eta_{Z}}$ is the inverse inertia coefficient (a mass parameter for the coordinate $\eta_{Z}$ ) (units: nucleon mass ${ }^{-1} \mathrm{fm}^{-2}$ ) and $V\left(\eta_{Z}\right)$ is the potential energy as a function of $\eta_{Z}$. This condenses to:

$$
\left[-\frac{\hbar^{2}}{2} \frac{\partial}{\partial \eta_{Z}}\left(B^{-1}\right)_{\eta_{Z} \eta_{Z}} \frac{\partial}{\partial \eta_{Z}}+V\left(\eta_{Z}\right)\right] \psi\left(\eta_{Z}\right)=E \psi\left(\eta_{Z}\right) .
$$

The inverse inertia coefficient is calculated using the equation [50]:

$$
\left(B^{-1}\right)_{\eta_{Z} \eta_{Z}}=\left(\frac{\partial \eta}{\partial \eta_{Z}}\right)^{-2}\left(B^{-1}\right)_{\eta \eta}
$$

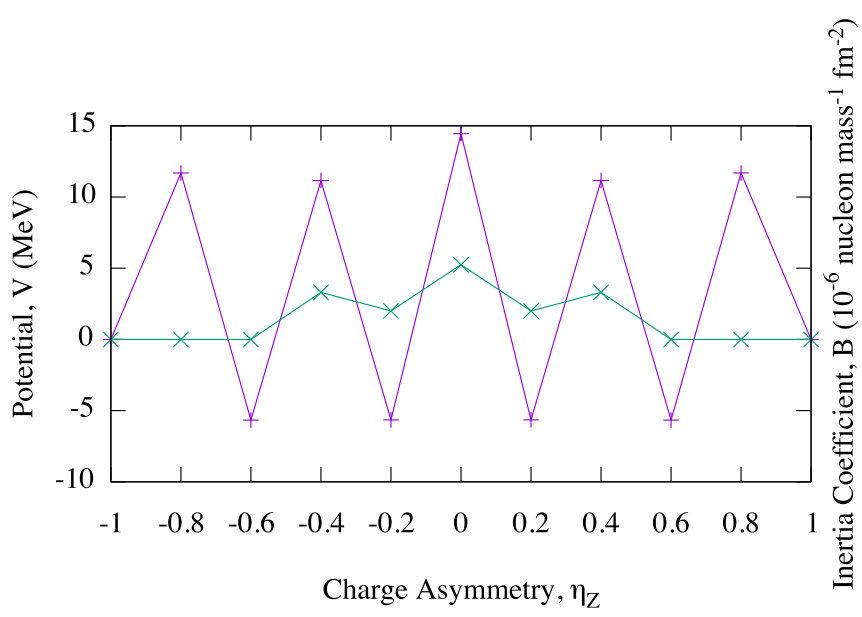

FIG. 3. Inertia coefficient $(\times) /$ potential $(+)$ plot for the fragmentation of ${ }_{10}^{20} \mathrm{Ne}$ in the charge asymmetry coordinate, with the potential energy of the compound nucleus $U 0=0 \mathrm{MeV}$. Markers denote values calculated, whilst intermediate values can be interpolated.

As in Ref. [55] $\left(B^{-1}\right)_{\eta \eta}$ is calculated using:

$$
\left(B^{-1}\right)_{\eta \eta}=\frac{1}{m_{0}} \frac{A_{\text {neck }}}{2 \sqrt{2 \pi} b^{2} A^{2}},
$$

where $A$ is the total nucleon number of the dinuclear system, $A_{\text {neck }}$ is the number of nucleons in the neck between the two fragments, $m_{0}$ is the nucleon mass and $b$ is a free parameter that characterizes the size of the neck. $A_{\text {neck }}$ is given by:

$$
A_{\text {neck }}=\int d \mathbf{r}\left[\rho_{1}(\mathbf{r})+\rho_{2}(\mathbf{r})\right] \exp \left(-\frac{\left(\mathbf{r}-\mathbf{r}_{0}\right)^{2}}{b^{2}}\right),
$$

where $\rho_{1}(\mathbf{r})$ and $\rho_{2}(\mathbf{r})$ are the nucleon densities of the two fragments as a function of the distance from the center of mass of the dinuclear system, $\mathbf{r}$, and $\mathbf{r}_{0}$ is the point where these two fragments densities are equal $\left[\rho_{1}\left(\mathbf{r}_{0}\right)=\rho_{2}\left(\mathbf{r}_{0}\right)\right]$. The neck here refers to the region of overlap between the intranuclear nucleon-distribution tails, as visualized by Fig. 2 .

$\eta$ (and by extension, $\eta_{Z}$ ) is treated as a continuous variable because at the touching configuration where the nuclei overlap, mass asymmetry (which is normally a discrete variable at larger distances) and the purely geometrical coordinate volume asymmetry (which is a continuous variable) are the same [10]. The dinuclear system is assumed to be filled by a continuous homogeneous fluid of mass and charge.

\section{Potentials}

The proposed solution to the time-independent Schrödinger equation postulates that each binary configuration of fragmentation has its own associated potential energy $V\left(\eta_{Z}\right)$, as exemplified by Fig. 3. For the potential, two spherical nuclei are considered at the touching configuration. These fragments have definite binding energies and interact through Coulomb and nuclear potentials. The total potential, $V$ is taken as the sum of the of the nuclear and Coulomb potentials, $V_{N}$ and $V_{C}$, respectively, in addition to the binding energies of the two fragments, $B E_{1}$ and $B E_{2}$, relative 
to the binding energy of the compound nucleus, $B E_{C N}$ :

$$
V=V_{N}+V_{C}+B E_{1}+B E_{2}-B E_{C N} .
$$

The strong variations in binding energy for different fragments give rise to the large energy fluctuations among the dinuclear configurations in Fig. 3. The inertia coefficient plot has been extrapolated from $\eta_{Z}= \pm 0.6$ to \pm 1 (due to insufficient data [56]), and so the inertia coefficient points for $\eta_{Z}= \pm 0.8$ are also expected to be local maxima. For the calculation of the mass parameter in the charge asymmetry coordinate, a macroscopic, geometrical model explained in Refs. [50,55] has been used.

The potentials in Fig. 3 were calculated using the BrogliaWinther approach [57], wherein the real part of the nucleusnucleus optical potential is assumed to have a Woods-Saxon shape:

$$
V_{N}(r)=-\frac{V_{0}}{1+\exp \left(\frac{r-R_{0}}{a}\right)},
$$

where

$$
V_{0}=16 \pi \frac{R_{1} R_{2}}{R_{1}+R_{2}} \gamma a, \quad a=0.63 \mathrm{fm},
$$

and

$$
R_{0}=R_{1}+R_{2}+0.29, \quad R_{i}=1.233 A_{i}^{1 / 3}-0.98 A_{i}^{-1 / 3},
$$

with surface energy constant $\gamma$ :

$$
\gamma=\gamma_{0}\left[1-k_{s}\left(\frac{N_{1}-Z_{1}}{A_{1}}\right)\left(\frac{N_{2}-Z_{2}}{A_{2}}\right)\right],
$$

where $\gamma_{0}$ and $k_{s}$ are assumed to be $0.95 \mathrm{MeV} / \mathrm{fm}^{2}$ and 1.8 , respectively. The Broglia-Winther potential has been used as a real nuclear potential to explain the elastic differential cross sections of many heavy-ion systems [57].

\section{Finite-difference method}

A finite-difference method solution to the timeindependent Schrödinger equation that incorporates periodic boundary conditions is presented in Ref. [58]. The premise of the method is to discretize the continuous variable (in this case, $x$ ) into a series of points with a finite difference $\Delta$ in such a manner that $x_{i}=x_{0}+i \Delta$, where $i$ is the step number $(i=0,1,2,3, \ldots, N)$.

Starting with the time-independent Schrödinger equation, the mass, $m$, is also taken as a function of the coordinate $x$ as well as the potential, $V$, and the wave function, $\psi$ [59]:

$$
-\frac{\hbar^{2}}{2} \frac{d}{d x}\left(\frac{1}{m(x)} \frac{d}{d x}\right) \psi(x)+V(x) \psi(x)=E \psi(x) .
$$

Taking $m(x)=m_{0} B(x)$ and multiplying by $\frac{2 m_{0}}{\hbar^{2}}$ :

$-\frac{d}{d x}\left(\frac{1}{B(x)} \frac{d}{d x} \psi(x)\right)+\frac{2 m_{0}}{\hbar^{2}} V(x) \psi(x)=\frac{2 m_{0}}{\hbar^{2}} E \psi(x)$.

Introducing the terms $v(x)=\frac{2 m_{0}}{\hbar^{2}} V(x)$ and $\mathcal{E}=\frac{2 m_{0}}{\hbar^{2}} E$ :

$$
-\frac{d}{d x}\left(\frac{1}{B(x)} \frac{d}{d x} \psi(x)\right)+v(x) \psi(x)=\mathcal{E} \psi(x) .
$$

Rewriting the derivatives using the finite difference method:

$$
-\frac{1}{\Delta x^{2}}\left(\frac{\psi_{i+1}-\psi_{i}}{B_{i+1 / 2}}-\frac{\psi_{i}-\psi_{i-1}}{B_{i-1 / 2}}\right)+v_{i} \psi_{i}=\mathcal{E} \psi_{i},
$$

where the intermediate points $(i \pm 1 / 2)$ are the mean values of the two adjacent points:

$$
B_{i+1 / 2}=\frac{1}{2}\left(B_{i+1}+B_{i}\right), \quad B_{i-1 / 2}=\frac{1}{2}\left(B_{i}+B_{i-1}\right) .
$$

Here the periodic boundary condition $\psi_{0}=\psi_{N}$ is enforced [60]. With this boundary condition the domain of definition of our wave function can formally be extended from the interval $x \in[0, L]$ to the whole number axis, with the requirement that the function be periodic with the period $L$ :

$$
\psi(x+L)=\psi(x) .
$$

The coordinate space $x$ is related to the charge asymmetry coordinate by:

$$
x=1-\eta_{Z},
$$

and as the range of $\eta_{Z}$ is 2, the range of $x$ [and therefore $L$ in Eq. (20)] must also be 2 .

In order to enforce this periodic boundary condition the tridiagonal Hamiltonian matrix must be amended by setting the top-right $(1, N)$ and bottom-left $(N, 1)$ elements equal to the subdiagonal elements, as per the matrix equation [Eq. (22)]:

$$
\begin{gathered}
\left(\begin{array}{ccccc}
-2 c+v_{1} & a & 0 & \cdots & a \\
b & -2 c+v_{2} & a & \cdots & 0 \\
0 & b & -2 c+v_{3} & \cdots & 0 \\
0 & 0 & b & \cdots & 0 \\
\vdots & \vdots & \vdots & \ddots & \vdots \\
b & 0 & 0 & \cdots & -2 c+v_{N}
\end{array}\right) \\
\times\left(\begin{array}{c}
\psi_{1} \\
\psi_{2} \\
\psi_{3} \\
\vdots \\
\psi_{N}
\end{array}\right)=\mathcal{E}\left(\begin{array}{c}
\psi_{1} \\
\psi_{2} \\
\psi_{3} \\
\vdots \\
\psi_{N}
\end{array}\right),
\end{gathered}
$$

where $v_{i}$ is the potential of the $i$ th step, $\mathcal{E}$ is the eigenenergy, $a=-\frac{1}{\Delta^{2}} \frac{1}{B_{i+1 / 2}}$ for elements above the diagonal, $b=-\frac{1}{\Delta^{2}} \frac{1}{B_{i-1 / 2}}$ for elements below the diagonal and $c=-\frac{1}{2}\left[\frac{1}{\Delta^{2}}\left(\frac{1}{B_{i+1 / 2}}+\frac{1}{B_{i-1 / 2}}\right)\right]$ for elements on the diagonal (for which $B$ is the mass parameter). Equation (22) is the practical application of Eq. (6) in the code. This tridiagonal matrix is then diagonalized by calling the LAPACK subroutine $d$ syevx [61], and the resultant eigenvectors are subsequently normalised so that the probability density function (PDF) $\psi^{2}$ can be computed and plotted as shown in Fig. 4.

In this example, ${ }_{10}^{20} \mathrm{Ne}$ is used as a test case because this nucleus has been used as a complex projectile in several ICF experiments [12-15]. Figures 4(a), 4(b), and 4(c) represent the PDF associated with the first, second, and third energy eigenvalues, respectively, for the fragmentation of ${ }_{10}^{20} \mathrm{Ne}$ in the 

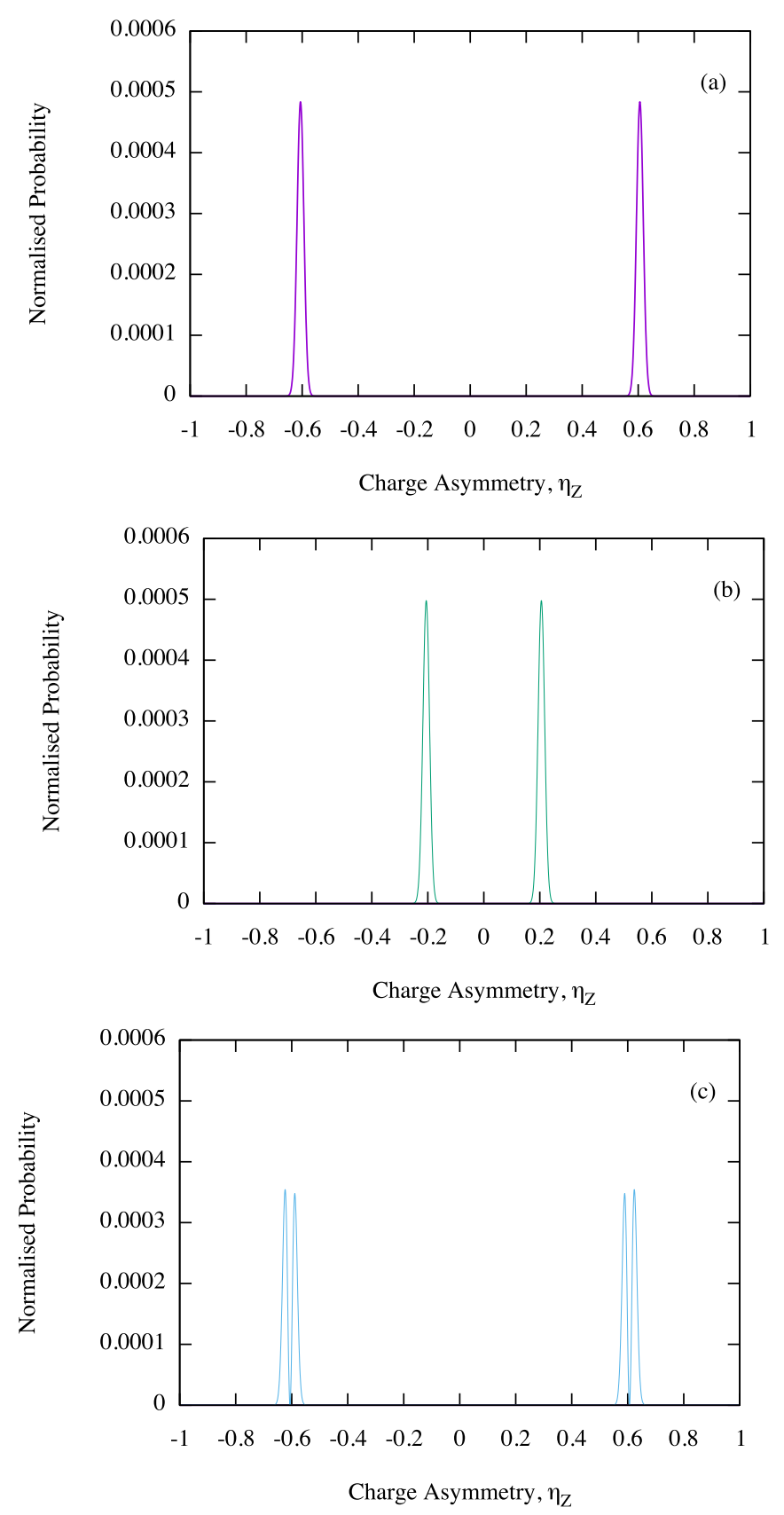

FIG. 4. Normalized PDF representing the fragmentation for the first three energy eigenvalues of ${ }_{10}^{20} \mathrm{Ne}$ in the charge asymmetry coordinate, with the potential energy and inertia coefficients of Fig. 3: (a) $-5.38 \mathrm{MeV}$, (b) $-5.34 \mathrm{MeV}$, and (c) $-4.76 \mathrm{MeV}$. The inertia coefficients and potentials of Fig. 3 have been interpolated here using $\Delta \eta_{Z}=0.001$.

charge asymmetry coordinate. These three energy eigenvalues correspond to six degenerate eigenvectors. This degeneracy arises from the symmetry of the potential about $\eta_{Z}=0$ in Fig. 4. For the first and third eigenvalues there are clear peaks at $\eta_{Z}= \pm 0.6$, which arise from fragment charges $Z_{1}$ and $Z_{2}$ of 8 and 2 , corresponding to ${ }_{8}^{16} \mathrm{O}$ and ${ }_{2}^{4} \mathrm{He}$. Similarly for the second eigenvalue there are clear peaks at $\eta_{Z}= \pm 0.2$, which arise from fragment charges $Z_{1}$ and $Z_{2}$ of 6 and 4 ,

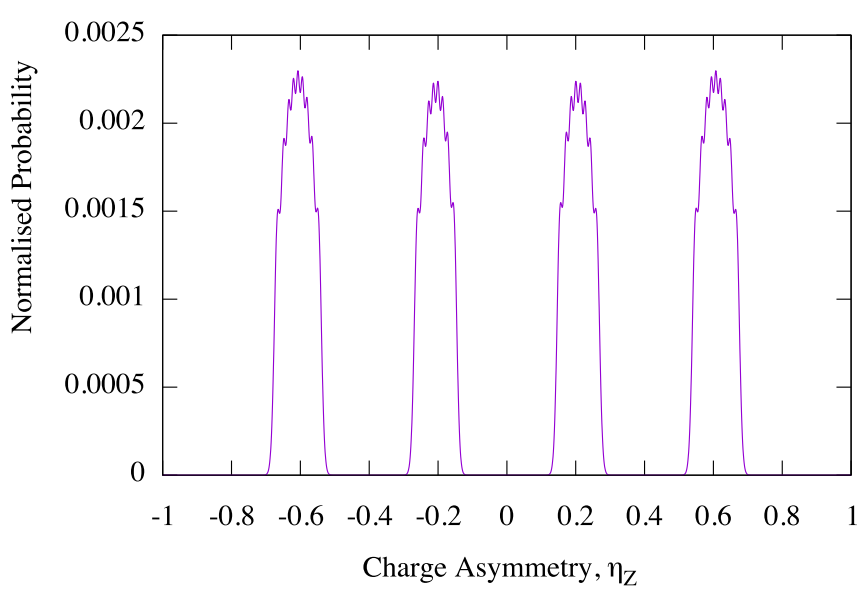

FIG. 5. Normalized total PDF representing the fragmentation for all 34 eigenvalues that lie within a $5 \mathrm{MeV}$ excitation energy range of ${ }_{10}^{20} \mathrm{Ne}$ in the charge asymmetry coordinate, with the potential energy and inertia coefficients of Fig. 3. The inertia coefficients and potentials of Fig. 3 have been interpolated here using $\Delta \eta_{Z}=0.001$.

corresponding to ${ }_{6}^{12} \mathrm{C}$ and ${ }_{4}^{8} \mathrm{Be}$. This shows that this method can successfully distinguish between the different binary configurations of fragmentation. The splitting of the peaks in Fig. 4(c) is due to the increased number of nodes in this higher excited state of ${ }_{10}^{20} \mathrm{Ne}$.

\section{E. Summing states}

A given projectile excitation energy range encompasses a certain number of eigenstates, and so in order to account for each state's contribution to the total fragmentation PDF they are summed using a Boltzmann factor [62]:

$$
\left|\Psi\left(\eta_{Z}\right)\right|^{2}=\frac{\sum_{i=0}^{N} e^{-E_{i} / T}\left|\psi_{i}\left(\eta_{Z}\right)\right|^{2}}{\sum_{i=0}^{N} e^{-E_{i} / T}},
$$

where $E_{i}$ is the eigenenergy of the $i$ th state and $T$ is the temperature in $\mathrm{MeV}$ given by:

$$
T=\sqrt{\frac{E_{\max }^{*}}{a}},
$$

where $E_{\max }^{*}$ is the maximum excitation energy of the projectile and the constant $a$ in $\mathrm{MeV}^{-1}$ is given by the Fermi gas model [62]:

$$
a=\frac{A_{P}}{10 \mathrm{MeV}}
$$

where $A_{P}$ is the nucleon number of the projectile nucleus.

The resulting total PDF for ${ }_{10}^{20} \mathrm{Ne}$ of Eq. (23) with a projectile excitation energy range of $5 \mathrm{MeV}$ is shown in Fig. 5. This PDF is then turned into a cumulative distribution function (CDF) and Monte Carlo sampled in order to select a binary fragmentation configuration. This is achieved via direct inversion of the CDF [63]. 

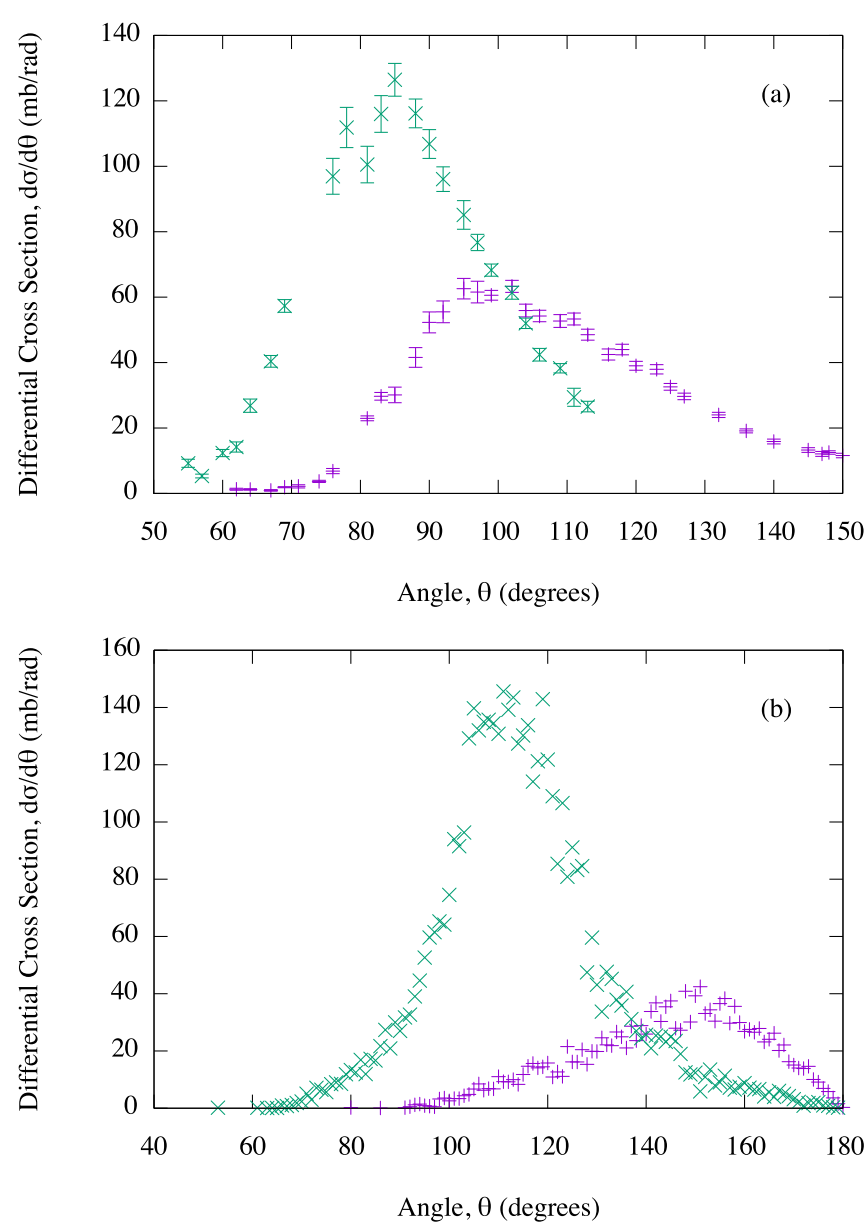

FIG. 6. Angular distributions for the transfer reaction of ${ }_{2}^{4} \mathrm{He}$ $\left({ }_{10}^{20} \mathrm{Ne}+{ }_{82}^{208} \mathrm{~Pb} \rightarrow{ }_{84}^{212} \mathrm{Po}+{ }_{8}^{16} \mathrm{O}\right)$ for projectile incident energies of $105 \mathrm{MeV}(+)$ and $115 \mathrm{MeV}(\times)$ : (a) from experiment [64], (b) from the present model calculations for a projectile with an excitation energy range $\left(E_{\max }^{*}-E_{\min }^{*}\right)$ of $1 \mathrm{keV}$.

\section{RESULTS AND DISCUSSION}

\section{A. Comparison with experimental data}

Experimental results [64] for the angular distribution for the transfer reaction of ${ }_{2}^{4} \mathrm{He}\left({ }_{10}^{20} \mathrm{Ne}+{ }_{82}^{208} \mathrm{~Pb} \rightarrow{ }_{84}^{212} \mathrm{Po}+{ }_{8}^{16} \mathrm{O}\right)$ for projectile incident energies of $105 \mathrm{MeV}$ and $115 \mathrm{MeV}$, collected using the ICARE charged particle multidetector array [65-67], are shown in Fig. 6(a). Results from the model presented in this paper for the angular distribution for the same reaction are presented in Fig. 6(b) using values for $\alpha$ and $\beta$ of 0.94 and 15 , respectively [see Eq. (1)]. These values of $\alpha$ and $\beta$ were selected because cross sections borne from them match experimental results closely. The position of the centroid of this angular distribution is sensitive to the value of $\alpha$; for example, in this case a value of $\alpha=0.74$ results in a peak shift of approximately -2 degrees. The magnitude of the resultant cross sections are sensitive to both $\alpha$ and $\beta$; in the same example where $\alpha=0.74$, the resultant cross sections are an order of magnitude greater. These values of $\alpha$ and $\beta$ were not chosen with the intention of perfectly matching the experimental results, but rather to demonstrate
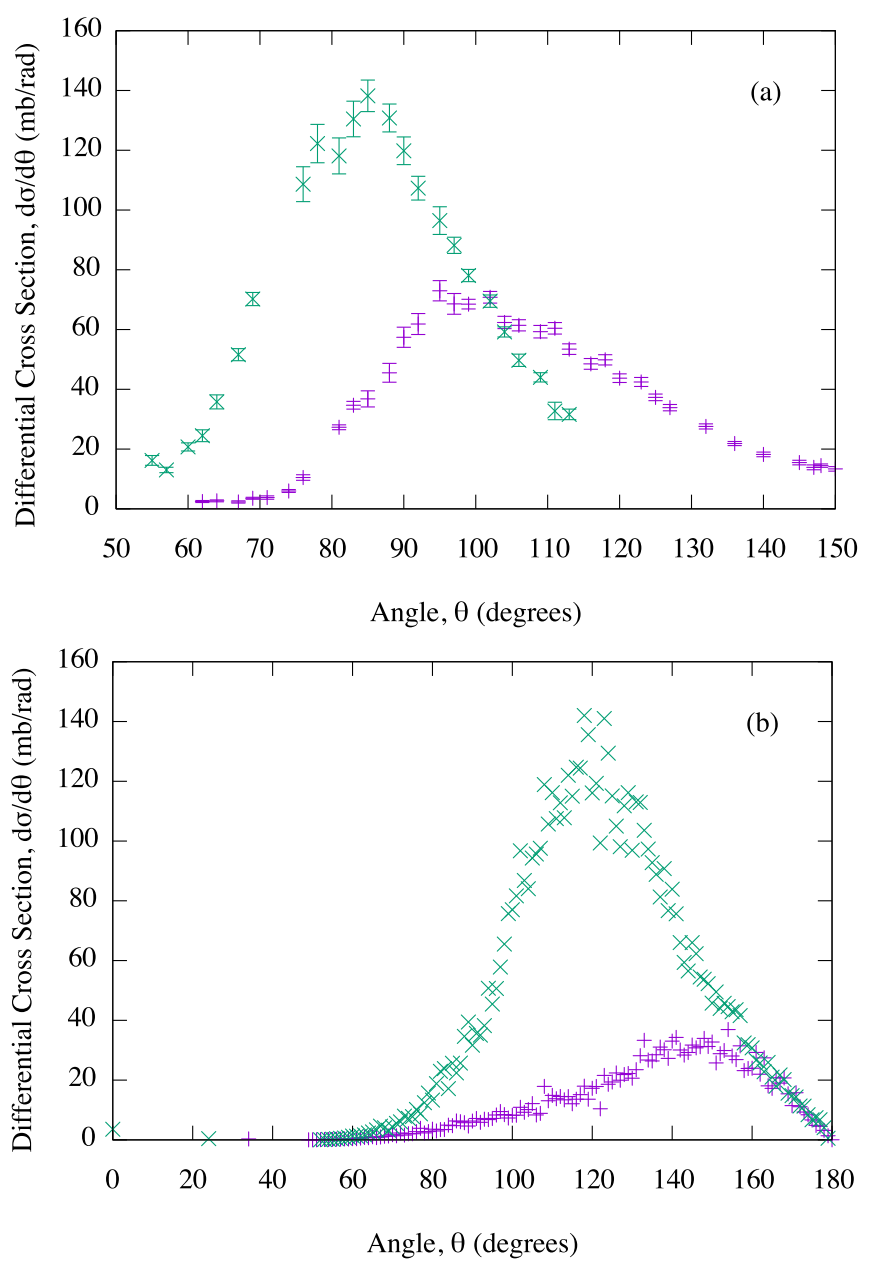

FIG. 7. Combined angular distributions for the transfer reactions of ${ }_{2}^{4} \mathrm{He}\left({ }_{10}^{20} \mathrm{Ne}+{ }_{82}^{208} \mathrm{~Pb} \rightarrow{ }_{84}^{212} \mathrm{Po}+{ }_{8}^{16} \mathrm{O}\right)$ and ${ }_{4}^{8} \mathrm{Be}\left({ }_{10}^{20} \mathrm{Ne}+{ }_{82}^{208} \mathrm{~Pb} \rightarrow\right.$ ${ }_{86}^{216} \mathrm{Rn}+{ }_{6}^{12} \mathrm{C}$ ) for projectile incident energies of $105 \mathrm{MeV}(+)$ and $115 \mathrm{MeV}(\times)$ : (a) from experiment [64], (b) from the present model calculations for a projectile with an excitation energy range $\left(E_{\max }^{*}-\right.$ $E_{\min }^{*}$ ) of $5 \mathrm{MeV}$.

a qualitative agreement. Figure 6(b) shows results for the reaction in the case where the projectile has an excitation energy range $\left(E_{\max }^{*}-E_{\min }^{*}\right)$ of $1 \mathrm{keV}$, which allows exclusively for the ${ }_{10}^{20} \mathrm{Ne}$ binary fragmentation combination of ${ }_{8}^{16} \mathrm{O}+{ }_{2}^{4} \mathrm{He}$. This allows for the direct comparison of the model results from Fig. 6(b) to the experimental results of Fig. 6(a), which for an incident energy $E_{0}=115 \mathrm{MeV}$ are of a very similar magnitude. For $E_{0}=105 \mathrm{MeV}$ the magnitudes of differential cross sections are less alike but still comparable. The main difference between these two figures is that the centroids of the distributions lie at vastly different angles, however, this can be explained as having arisen due to the global BrogliaWinther potential used to simulate the interaction between the three bodies.

Figure 7(a) contains experimental results [64] for the combined angular distribution for the transfer reactions of ${ }_{2}^{4} \mathrm{He}$ $\left.{ }_{10}^{20} \mathrm{Ne}+{ }_{82}^{208} \mathrm{~Pb} \rightarrow{ }_{84}^{212} \mathrm{Po}+{ }_{8}^{16} \mathrm{O}\right)$ and ${ }_{4}^{8} \mathrm{Be}\left({ }_{10}^{20} \mathrm{Ne}+{ }_{82}^{208} \mathrm{~Pb} \rightarrow\right.$ ${ }_{86}^{216} \mathrm{Rn}+{ }_{6}^{12} \mathrm{C}$ ) for projectile incident energies of $105 \mathrm{MeV}$ and $115 \mathrm{MeV}$, while Fig. 7(b) contains results from the present 


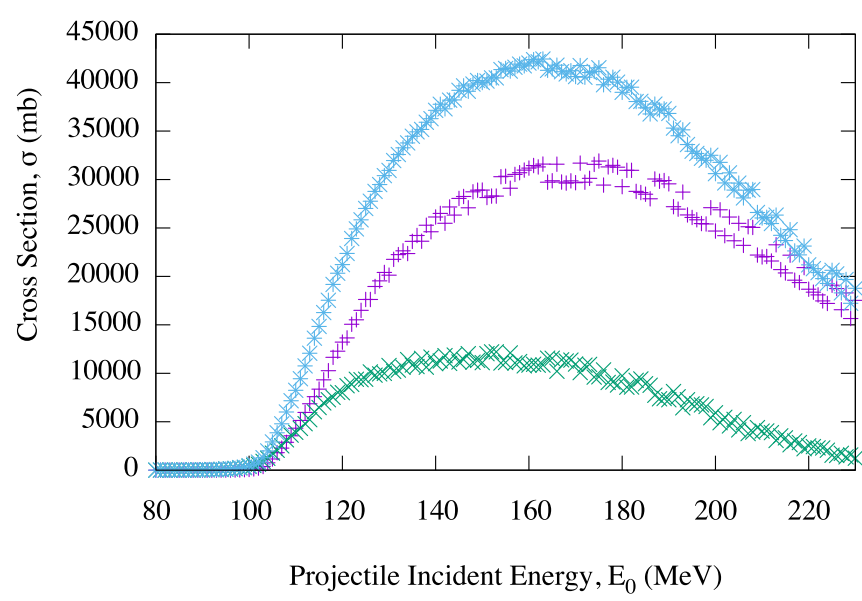

FIG. 8. ICF cross sections for ICF channels $1(+), 2(\times)$ and both combined $(*)$ for the reaction ${ }_{10}^{20} \mathrm{Ne}+{ }_{82}^{208} \mathrm{~Pb}$ for a projectile with an excitation energy range $\left(E_{\max }^{*}-E_{\min }^{*}\right)$ of $5 \mathrm{MeV}$, for a range of projectile incident energies $\left(E_{0}\right)$. ICF channel 1 refers to the production of ${ }_{90}^{224} \mathrm{Th}$ and ${ }_{88}^{220} \mathrm{Ra}$, while ICF channel 2 refers to the production of ${ }_{84}^{212} \mathrm{Po}$ and ${ }_{86}^{216} \mathrm{Rn}$.

model for the combined angular distribution for the same reactions, also using values for $\alpha$ and $\beta$ of 0.94 and 15, respectively. Figure $7(\mathrm{~b})$ shows results for the reaction in the case where the projectile has an excitation energy range $\left(E_{\max }^{*}-E_{\min }^{*}\right)$ of $5 \mathrm{MeV}$, which allows for the ${ }_{10}^{20} \mathrm{Ne}$ binary fragmentation combination of not only ${ }_{8}^{16} \mathrm{O}+{ }_{2}^{4} \mathrm{He}$ but also that of ${ }_{6}^{12} \mathrm{C}+{ }_{4}^{8} \mathrm{Be}$, permitting direct comparison with the experimental results of Fig. 7(a). As with the Fig. 6 comparison, the differential cross sections of Fig. 7 are of a very similar magnitude for an incident energy of $E_{0}=115 \mathrm{MeV}$ but less so for $E_{0}=105 \mathrm{MeV}$, yet still comparable, and again the main difference between these two figures is that the cross sections are obtained at vastly different angles.

As the present semiclassical model is based on a breakupfusion picture, it cannot treat the quantum-mechanical $\alpha$ stripping process [68], which might also play a part in explaining the discrepancies between the data of Figs. 6(a) and 6(b) and the data of Figs. 7(a) and 7(b). Taking all the aforementioned into consideration, these comparisons indicate that this new model is indeed accurate for the purpose of determining how a projectile nucleus will fragment and subsequently undergo ICF with a target.

\section{B. Total ICF cross sections}

Figure 8 presents results from the model for ICF cross sections for the reaction ${ }_{10}^{20} \mathrm{Ne}+{ }_{82}^{208} \mathrm{~Pb}$ for a range of projectile incident energies $\left(E_{0}\right)$, with a projectile excitation energy range of $5 \mathrm{MeV}$. An excitation energy range of $1 \mathrm{keV}$ permits only the binary fragmentation combination of ${ }_{8}^{16} \mathrm{O}+{ }_{2}^{4} \mathrm{He}$ from ${ }_{10}^{20} \mathrm{Ne}$, whereas a $5 \mathrm{MeV}$ excitation energy range also permits the binary fragmentation combination of ${ }_{6}^{12} \mathrm{C}+{ }_{4}^{8} \mathrm{Be}$. Thus, ICF channel 1 includes the independent capture of ${ }_{8}^{16} \mathrm{O}$ and ${ }_{6}^{12} \mathrm{C}$ by the target ${ }_{8}^{16} \mathrm{O}+{ }_{82}^{208} \mathrm{~Pb} \rightarrow{ }_{90}^{224} \mathrm{Th},{ }_{6}^{12} \mathrm{C}+{ }_{82}^{208} \mathrm{~Pb} \rightarrow$ ${ }_{88}^{220} \mathrm{Ra}$ ), while ICF channel 2 includes the independent capture of ${ }_{2}^{4} \mathrm{He}$ and ${ }_{4}^{8} \mathrm{Be}$ by the target ${ }_{2}^{4} \mathrm{He}+{ }_{82}^{208} \mathrm{~Pb} \rightarrow{ }_{84}^{212} \mathrm{Po},{ }_{4}^{8} \mathrm{Be}+$
${ }_{82}^{208} \mathrm{~Pb} \rightarrow{ }_{86}^{216} \mathrm{Rn}$ ). A plot of cross sections summed from both ICF channels is also included in Fig. 8.

The results in Fig. 8 are also for a projectile with an excitation energy range of $5 \mathrm{MeV}$, and this was expressly chosen so that multiple binary fragmentation combinations would have to be handled by the model, so as to showcase its capabilities.

Figure 8 suggests that for the reaction ${ }_{10}^{20} \mathrm{Ne}+{ }_{82}^{208} \mathrm{~Pb}$ with a projectile excitation energy $E^{*}=5 \mathrm{MeV}$, the projectile incident energy $E_{0} \approx 150 \mathrm{MeV}$ yields the greatest probability for the ICF of either the ${ }_{8}^{16} \mathrm{O}$ or ${ }_{6}^{12} \mathrm{C}$ fragments with the target, $E_{0} \approx 170 \mathrm{MeV}$ yields the greatest probability for the ICF of either the ${ }_{2}^{4} \mathrm{He}$ or ${ }_{4}^{8} \mathrm{Be}$ fragments with the target, and $E_{0} \approx$ $160 \mathrm{MeV}$ yields the greatest probability for the ICF of any fragment with the target.

\section{ICF distributions}

As with the PLATYPUS code, this model can also be used to calculate angular distributions, angular momentum distribu-
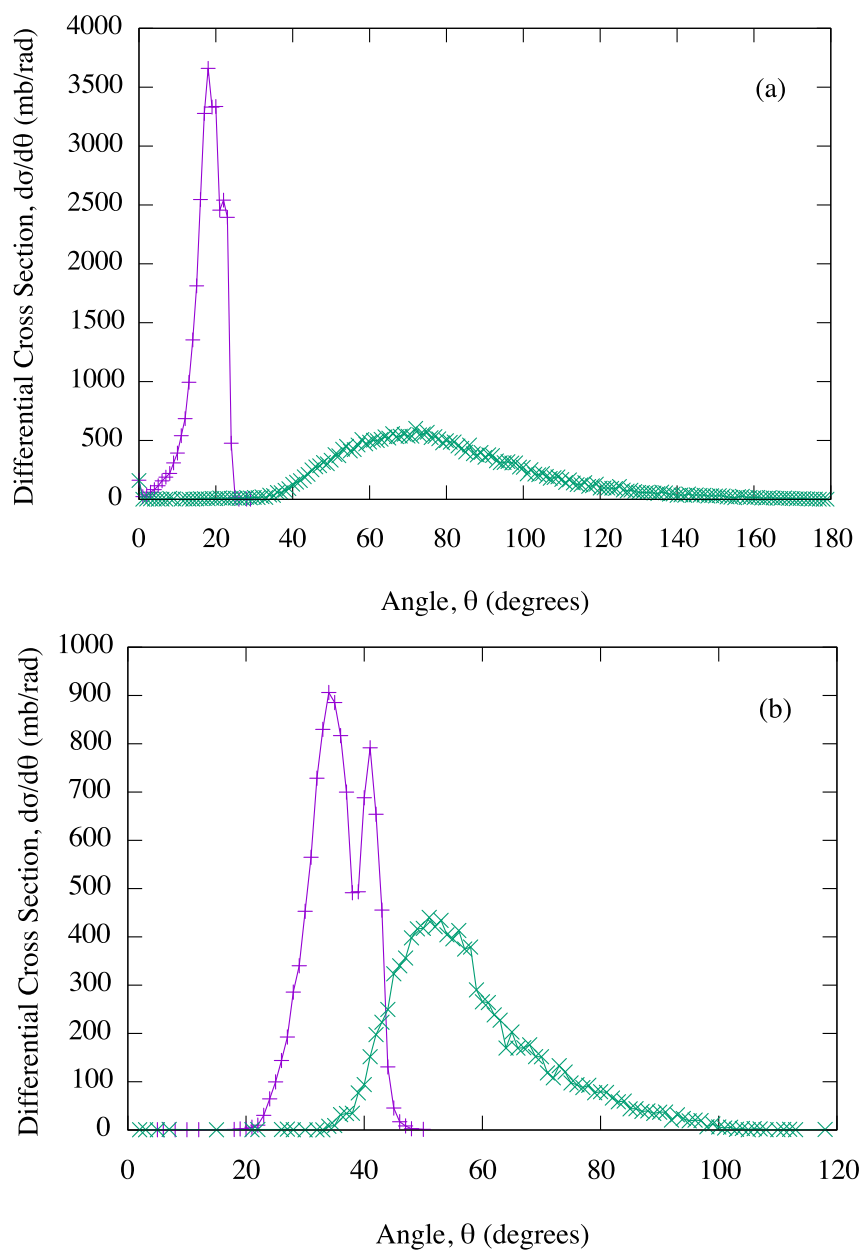

FIG. 9. Inclusive angular distributions of ICF products $(+)$ and associated survival fragments $(\times)$ for the reaction ${ }_{10}^{20} \mathrm{Ne}+{ }_{82}^{208} \mathrm{~Pb}$ for a projectile with an excitation energy range $\left(E_{\max }^{*}-E_{\min }^{*}\right)$ of $5 \mathrm{MeV}$, for a projectile incident energy $\left(E_{0}\right)$ of $160 \mathrm{MeV}$ : (a) ICF channel 1 $\left({ }_{90}^{224} \mathrm{Th}\right.$ and $\left.{ }_{88}^{220} \mathrm{Ra}\right)$, (b) ICF channel $2\left({ }_{84}^{212} \mathrm{Po}\right.$ and $\left.{ }_{86}^{216} \mathrm{Rn}\right)$. 


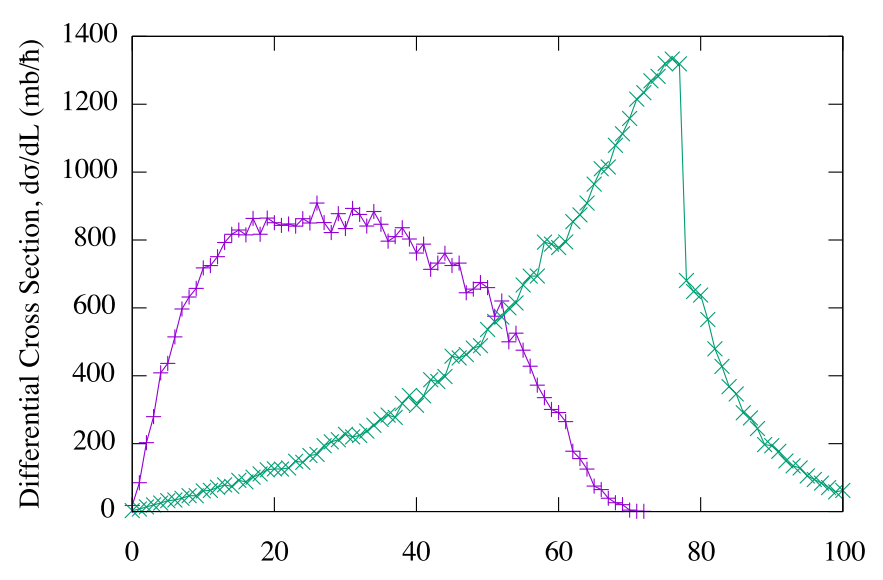

Angular Momentum, L ( $)$

FIG. 10. Angular momentum distributions of the inclusive ICF products (the sum of all the ICF channels) for the same reaction as in Fig. 9, using two different representations, namely in terms of the angular momentum brought by the ICF fragments into the target $(+)$ and the ${ }_{10}^{20} \mathrm{Ne}$ orbital angular momentum $(\times)$.

tions, excitation energy distributions and asymptotic angular distributions as shown in Figs. 9, 10, 11, and 12, respectively, for any ICF channels relevant to the projectile and its potential binary fragmentation combinations.

Figure 9 presents the ICF angular distributions for the same reaction as in the previous subsection, but for a projectile incident energy $\left(E_{0}\right)$ of only $160 \mathrm{MeV}$ rather than for a whole range of projectile incident energies. $160 \mathrm{MeV}$ was chosen as an area of interest because this energy is approximately where the peaks from both ICF channels overlap in Fig. 8. Similarly, Fig. 10 presents the ICF spin distributions, Fig. 11 presents the excitation energy distribution of the primary ICF products (channel 1: ${ }_{90}^{224} \mathrm{Th}$ and ${ }_{88}^{220} \mathrm{Ra}$, channel 2: ${ }_{84}^{212} \mathrm{Po}$ and ${ }_{86}^{216} \mathrm{Rn}$ ), and Fig. 12 presents the asymptotic angular distributions of the

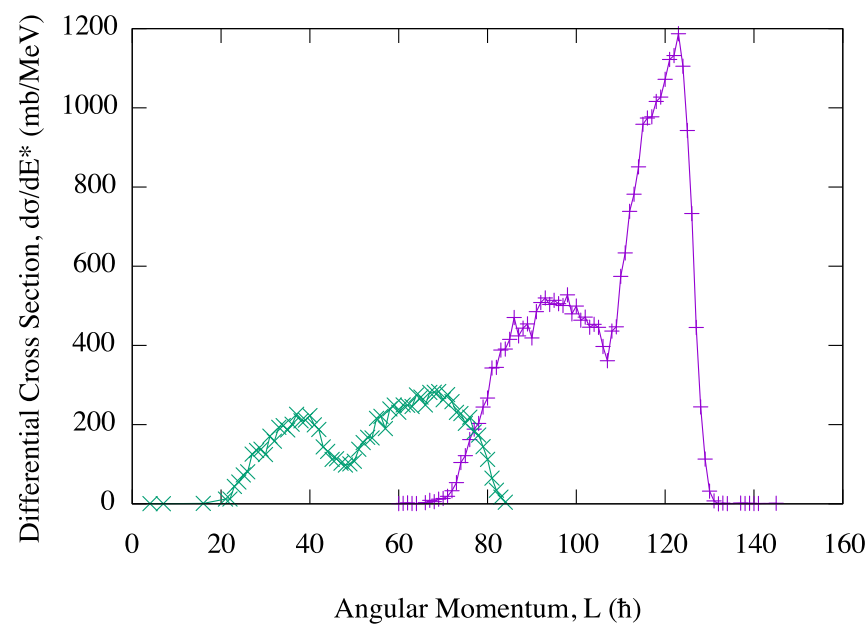

FIG. 11. Excitation energy distribution of the primary ICF products for ICF channels $1(+)$ and $2(\times)$ for the same reaction as in Fig. 9. ICF channel 1 refers to the production of ${ }_{90}^{224} \mathrm{Th}$ and ${ }_{88}^{220} \mathrm{Ra}$, while ICF channel 2 refers to the production of ${ }_{84}^{212} \mathrm{Po}$ and ${ }_{86}^{216} \mathrm{Rn}$.

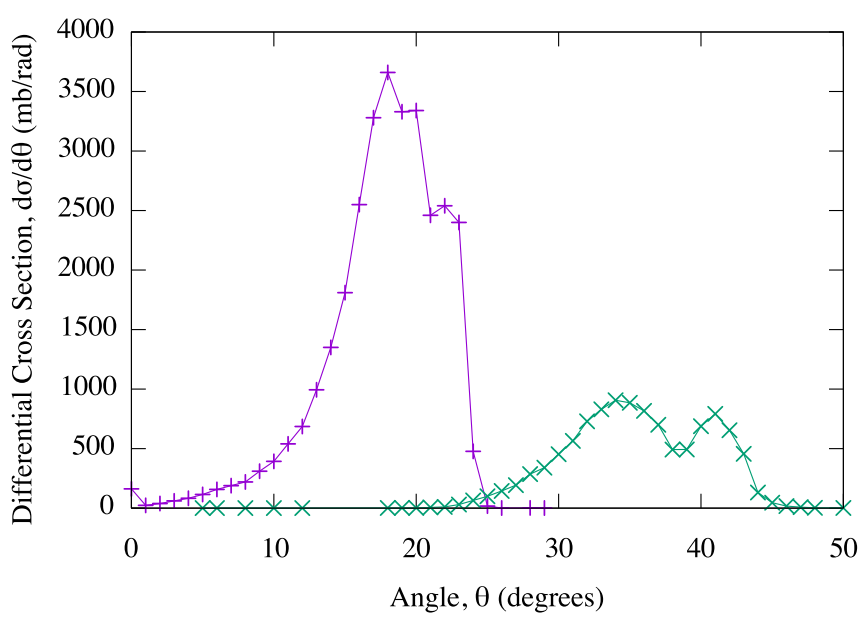

FIG. 12. Asymptotic angular distribution of the ICF products and the surviving breakup fragments for ICF channels $1(+)$ and $2(\times)$ for the same reaction as in Fig. 9. ICF channel 1 refers to the production of ${ }_{90}^{224} \mathrm{Th}$ and ${ }_{88}^{220} \mathrm{Ra}$, while ICF channel 2 refers to the production of ${ }_{84}^{212} \mathrm{Po}$ and ${ }_{86}^{216} \mathrm{Rn}$.

ICF products (channel 1: ${ }_{90}^{224} \mathrm{Th}$ and ${ }_{88}^{220} \mathrm{Ra}$, channel 2: ${ }_{84}^{212} \mathrm{Po}$ and ${ }_{86}^{216} \mathrm{Rn}$ ) and the surviving breakup fragments (channel 1 : ${ }_{2}^{4} \mathrm{He}$ and ${ }_{4}^{8} \mathrm{Be}$, channel 2: ${ }_{8}^{16} \mathrm{O}$ and ${ }_{6}^{12} \mathrm{C}$ ).

For the reaction ${ }_{10}^{20} \mathrm{Ne}+{ }_{82}^{208} \mathrm{~Pb}$ with projectile excitation energy $E^{*}=5 \mathrm{MeV}$ and projectile incident energy $E_{0}=$ $160 \mathrm{MeV}$, the angular distributions shown in Fig. 9(a) suggest that the highest cross section yields for the ICF products ${ }_{90}^{224} \mathrm{Th}$ and ${ }_{88}^{220} \mathrm{Ra}$ can be found at angles of approximately $17-23$ degrees, and the highest yields for the associated survival fragments ${ }_{2}^{4} \mathrm{He}$ and ${ }_{4}^{8} \mathrm{Be}$ can be found at angles of approximately 60-80 degrees. Similarly, for the same reaction, the angular distributions shown in Fig. 9(b) suggest that the highest crosssection yields for the ICF products ${ }_{84}^{212} \mathrm{Po}$ and ${ }_{86}^{216} \mathrm{Rn}$ can be found at angles of approximately 30-43 degrees, and the highest yields for the associated survival fragments ${ }_{8}^{16} \mathrm{O}$ and ${ }_{6}^{12} \mathrm{C}$ can be found at angles of approximately $43-63$ degrees.

The angular momentum distributions shown in Fig. 10 suggest that the highest ICF cross section yields can be found at angular momenta of approximately $7-52 \hbar$ concerning the angular momentum brought by the ICF fragments $\left({ }_{8}^{16} \mathrm{O},{ }_{6}^{12} \mathrm{C}\right.$, ${ }_{4}^{8} \mathrm{Be}$, and $\left.{ }_{2}^{4} \mathrm{He}\right)$ into the target $\left({ }_{82}^{208} \mathrm{~Pb}\right)$, and at angular momenta of approximately $55-80 \hbar$ concerning the projectile $\left({ }_{10}^{20} \mathrm{Ne}\right)$ orbital angular momentum.

The excitation energy distributions shown in Fig. 11 suggest that of the primary ICF products ${ }_{90}^{224} \mathrm{Th}$ and ${ }_{88}^{220} \mathrm{Ra}$, those with excitation energies of approximately $80-128 \mathrm{MeV}$ have the highest cross section yields, while of the primary ICF products ${ }_{84}^{212} \mathrm{Po}$ and ${ }_{86}^{216} \mathrm{Rn}$, those with excitation energies of approximately $27-80 \mathrm{MeV}$ have the highest cross section yields. It is clear that for each of these ICF channels there are two distinct peaks; for ICF channel 1 one likely arises from ${ }_{90}^{224} \mathrm{Th}$ and the other from ${ }_{88}^{220} \mathrm{Ra}$, similarly for ICF channel 2 one likely arises from ${ }_{84}^{212} \mathrm{Po}$ and the other from ${ }_{86}^{216} \mathrm{Rn}$.

The asymptotic angular distributions shown in Fig. 12 suggest that the highest cross-section yields for ICF products 
${ }_{90}^{224} \mathrm{Th}$ and ${ }_{88}^{220} \mathrm{Ra}$ and their surviving breakup fragments ${ }_{2}^{4} \mathrm{He}$ and ${ }_{4}^{8} \mathrm{Be}$ can be found at angles of approximately $14-24$ degrees, while the highest cross-section yields for ICF products ${ }_{84}^{212} \mathrm{Po}$ and ${ }_{86}^{216} \mathrm{Rn}$ and their surviving breakup fragments ${ }_{8}^{16} \mathrm{O}$ and ${ }_{6}^{12} \mathrm{C}$ can be found at angles of approximately $30-43$ degrees.

\section{General remarks}

All the information provided by the present model is also very useful for planning dedicated experimental setups for production of specific isotopes through the ICF reaction mechanism. A limitation of the current implementation of the model is that there are only two ICF channels in the computation: one for the ICF of the heavier fragment and target, and one for the ICF of the lighter fragment and target. The next major step in this model's development is the disentanglement of all the individual ICF channels. For example, in the case where a projectile has an excitation energy range of $5 \mathrm{MeV}$, as presented in Fig. 8, there ought to be four ICF channels: one for the ICF of ${ }_{8}^{16} \mathrm{O}$ and the target, one for the ICF of ${ }_{6}^{12} \mathrm{C}$ and the target, one for the ICF of ${ }_{4}^{8} \mathrm{Be}$ and target, and one for the ICF of ${ }_{2}^{4} \mathrm{He}$ and the target. This will allow for more universal input to the model and much more informative output from it. Additional steps include further refinements of the model, so as to make it as accurate and as true to life as possible.

\section{CONCLUSIONS}

In summary, the ICF dynamics of ${ }_{10}^{20} \mathrm{Ne}+{ }_{82}^{208} \mathrm{~Pb}$ collisions at energies above the Coulomb barrier have been investigated using a novel semiclassical dynamical model, built upon a classical trajectory model with stochastic breakup, featuring a dynamical fragmentation theory treatment of two-body clusterization and decay of a projectile. This has been implemented via a finite-difference method solution to the time-independent Schrödinger equation in the charge asymmetry coordinate by diagonalizing a tridiagonal Hamiltonian matrix with periodic boundary conditions. Initial results look promising and indicate that this method can be a successful way of probing ICF of complex nuclei at Coulomb energies, and once the model has been sufficiently polished following disentanglement of the ICF channels and other minor refinements it will likely prove to be an invaluable tool in making predictions for the formation of SHEs through the ICF reaction mechanism.

\section{ACKNOWLEDGMENTS}

We thank Professor Nikolai Antonenko and Professor Gurgen Adamian for useful discussions and constructive comments, Ivan Rogov for providing the inverse inertia coefficient shown in Fig. 3, and Yusuf El-Hassan for assistance with extracting data in tabular form from figures in Ref. [64]. The support from the STFC grant (ST/P005314/1) is acknowledged.
[1] H. Kragh, From Transuranic to Superheavy Elements: A Story of Dispute and Creation (Springer, Berlin, 2018).

[2] A. Sobiczewski, F. A. Gareev, and B. N. Kalinkin, Phys. Lett. 22, 500 (1966).

[3] Y. T. Oganessian, V. K. Utyonkov, Y. V. Lobanova, F. S. Abdullin, A. N. Polyakov, I. V. Shirokovsky, Y. S. Tsyganov, G. G. Gulbekian, S. L. Bogomolov et al., Nucl. Phys. A 734, 109 (2004).

[4] G. T. Seaborg and W. D. Loveland, The Elements Beyond Uranium (Wiley-Interscience, New York, 1990).

[5] S. Ćwiok, J. Dobaczewski, P.-H. Heenen, P. Magierski, and W. Nazarewicz, Nucl. Phys. A 611, 211 (1996).

[6] S. G. Nilsson, C. F. Tsang, A. Sobiczewski, Z. Szymański, S. Wycech, C. Gustafson, I.-L. Lamm, P. Möller, and B. Nilsson, Nucl. Phys. A 131, 1 (1969).

[7] U. Mosel and W. Greiner, Z. Phys. 217, 256 (1968); 222, 261 (1969).

[8] S. Hofmann and G. Münzenberg, Rev. Mod. Phys. 72, 733 (2000).

[9] P. Lichtner, D. Dreschsel, J. Maruhn, and W. Greiner, Phys. Lett. B 45, 175 (1973).

[10] H. J. Fink, J. Maruhn, W. Scheid, and W. Greiner, Z. Phys. 268, 321 (1974).

[11] O. Zohni, J. Maruhn, W. Scheid, and W. Greiner, Z. Phys. 275, 235 (1975).

[12] C. Borcea, E. Gierlik, R. Kalpakchieva, Y. T. Oganessian, and Y. E. Penionzhkevich, Nucl. Phys. A 351, 312 (1981).
[13] C. Borcea, E. Gierlik, A. M. Kalinin, R. Kalpakchieva, Y. T. Oganessian, T. Pawlat, Y. E. Penionzhkevich, and A. V. Rykhlyuk, Nucl. Phys. A 391, 520 (1982).

[14] C. Borcea, E. Gierlik, R. Kalpakchieva, N. H. Chau, Y. T. Oganessian, T. Pawlat, and Y. E. Penionzhkevich, Nucl. Phys. A 415, 169 (1984).

[15] H. Homeyer, U. Jahnke, G. Ingold, M. Bürgel, H. Fuchs, and D. Hilscher, Z. Phys. A - Atoms and Nuclei 314, 143 (1983).

[16] H. C. Britt and A. R. Quinton, Phys. Rev. 124, 877 (1961).

[17] J. Galin, B. Gatty, D. Guerreau, C. Rousset, U. C. SchlotthauerVoos, and X. Tarrago, Phys. Rev. C 9, 1126 (1974).

[18] T. Udagawa and T. Tamura, Phys. Rev. Lett. 45, 1311 (1980).

[19] J. R. Wu and I. Y. Lee, Phys. Rev. Lett. 45, 8 (1980).

[20] J. R. Wu, C. C. Chang, and H. D. Holmgren, Phys. Rev. Lett. 40, 1013 (1978).

[21] J. Wilczyński, K. Siwek-Wilczyńska, J. van Driel, S. Gonggrijp, D. C. J. M. Hageman, R. V. F. Janssens, J. Łukasiak, and R. H. Siemssen, Phys. Rev. Lett. 45, 606 (1980).

[22] I. M. Brâncuş, H. Rebel, J. Wentz, and V. Corcalciuc, Phys. Rev. C 42, 2157 (1990).

[23] J. P. Bondorf, J. N. De, G. Fái, A. O. T. Karvinen, B. Jakobsson, and J. Randrup, Nucl. Phys. A 333, 285 (1980).

[24] D. H. E. Gross and J. Wilczyński, Phys. Lett. B 67, 1 (1977).

[25] H. Tricoire, C. Gerschel, A. Gillibert, and N. Perrin, Z. Phys. A 323, 163 (1986).

[26] T. C. Awes, G. Poggi, C. K. Gelbke, B. B. Back, B. G. Glagola, H. Breuer, and V. E. Viola, Jr., Phys. Rev. C 24, 89 (1981).

[27] M. Blann, Phys. Rev. C 23, 205 (1981). 
[28] T. Otsuka and K. Harada, Phys. Lett. B 121, 106 (1983).

[29] B. G. Harvey, Nucl. Phys. A 444, 498 (1985).

[30] M. H. Simbel and A. Y. Abul-Magd, Z. Phys. A 294, 277 (1980).

[31] W. W. Morison, S. K. Samaddar, D. Sperber, and M. ZielińskaPfabé, Phys. Lett. B 99, 205 (1981).

[32] K. Möhring, T. Srokowski, D. H. E. Gross, and H. Homeyer, Phys. Lett. B 203, 210 (1988).

[33] H. Morgenstern, W. Bohne, W. Galster, K. Grabisch, and A. Kyanowski, Phys. Rev. Lett. 52, 1104 (1984).

[34] U. Gupta, P. P. Singh, D. P. Singh, M. K. Sharma, A. Yadav, R. Kumar, B. P. Singh, and R. Pras, Nucl. Phys. A 811, 77 (2008).

[35] P. P. Singh, B. P. Singh, M. K. Sharma, U. Gupta, D. P. Singh, R. Prasad, R. Kumar, and K. S. Golda, Phys. Rev. C 77, 014607 (2008).

[36] T. Inamura, M. Ishihara, T. Fukuda, T. Shimoda, and H. Hiruta, Phys. Lett. B 68, 51 (1977).

[37] T. Inamura, T. Kojima, T. Nomura, T. Sugitate, and H. Utsunomiya, Phys. Lett. B 84, 71 (1979).

[38] T. Inamura, A. C. Kahler, D. R. Zolnowski, U. Garg, T. T. Sugihara, and M. Wakai, Phys. Rev. C 32, 1539 (1985).

[39] D. R. Zolnowski, H. Yamada, S. E. Cala, A. C. Kahler, and T. T. Sugihara, Phys. Rev. Lett. 41, 92 (1978).

[40] K. A. Geoffroy, D. G. Sarantites, M. L. Halbert, D. C. Hensley, R. A. Dayras, and J. H. Barker, Phys. Rev. Lett. 43, 1303 (1979).

[41] W. Trautmann, O. Hansen, H. Tricoire, W. Hering, R. Ritzka, and W. Trombik, Phys. Rev. Lett. 53, 1630 (1984).

[42] C. Gerschel, Nucl. Phys. A 387, 297 (1982).

[43] R. L. Robinson, R. L. Auble, I. Y. Lee, M. J. Martin, G. R. Young, J. Gomez del Campo, J. B. Ball, F. E. Bertrand, R. L. Ferguson et al., Phys. Rev. C 24, 2084 (1981).

[44] H. Utsunomiya, T. Nomura, M. Ishihara, T. Sugitate, K. Ieki, and S. Kohmoto, Phys. Lett. B 105, 135 (1981).

[45] J. H. Barker, J. R. Beene, M. L. Halbert, D. C. Hensley, M. Jääskeläinen, D. G. Sarantites, and R. Woodward, Phys. Rev. Lett. 45, 424 (1980).

[46] B. B. Back, H. Esbensen, C. L. Jiang, and K. E. Rehm, Rev. Mod. Phys. 86, 317 (2014).

[47] L. F. Canto, P. R. S. Gomes, R. Donangelo, J. Lubian, and M. S. Hussein, Phys. Rep. 596, 1 (2015).

[48] I. Stefan, B. Fornal, S. Leoni, F. Azaiez, C. Portail, J. C. Thomas, A. V. Karpov, D. Ackermann, P. Bednarczyk et al., Phys. Lett. B 779, 456 (2018).
[49] A. Diaz-Torres, J. Phys. G 37, 075109 (2010); Comput. Phys. Commun. 182, 1100 (2011).

[50] S. N. Kuklin, T. M. Shneidman, G. G. Adamian, and N. V. Antonenko, Eur. Phys. J. A 48, 112 (2012).

[51] A. Diaz-Torres, D. J. Hinde, J. A. Tostevin, M. Dasgupta, and L. R. Gasques, Phys. Rev. Lett. 98, 152701 (2007).

[52] D. J. Hinde, M. Dasgupta, B. R. Fulton, C. R. Morton, R. J. Wooliscroft, A. C. Berriman, and K. Hagino, Phys. Rev. Lett. 89, 272701 (2002).

[53] K. J. Cook, E. C. Simpson, D. H. Luong, S. Kalkal, M. Dasgupta, and D. J. Hinde, Phys. Rev. C 93, 064604 (2016).

[54] S. Kalkal, E. C. Simpson, D. H. Luong, K. J. Cook, M. Dasgupta, D. J. Hinde, I. P. Carter, D. Y. Jeung, G. Mohanto et al., Phys. Rev. C 93, 044605 (2016).

[55] G. G. Adamian, N. V. Antonenko, and R. V. Jolos, Nucl. Phys. A 584, 205 (1995).

[56] G. G. Adamian (private communication).

[57] W. Reisdorf, J. Phys. G 20, 1297 (1994).

[58] Y. Murayama, Mesoscopic Systems: Fundamentals and Applications (Wiley, New York, 2001), Appendix A.

[59] J. D. Cooper, A. Valavanis, Z. Ikonić, P. Harrison, and J. E. Cunningham, J. Appl. Phys. 108, 113109 (2010).

[60] B. V. Svistunov, Periodic Boundary Conditions. Classical Limit, University of Massachusetts Amherst. Available at: http://people.umass.edu/bvs/pbc.pdf.

[61] LAPACK Linear Algebra PACKage (updated 2017). Available at: www.netlib.org/lapack/.

[62] W. Greiner, J. Y. Park, and W. Scheid, Nuclear Molecules (World Scientific Publishing, Singapore, 1994).

[63] R. L. Harrison, AIP Conf. Proc. 1204, 17 (2010).

[64] I. Strojek, W. Czarnacki, W. Gawlikowicz, N. Keeley, M. Kisieliński, S. Kliczewski, A. Kordyasz, E. Koshchiy, M. Kowalczyk et al., HIL Annual Report 2010, 37-38, 2010.

[65] C. Bhattacharya, M. Rousseau, C. Beck, V. Rauch, R. M. Freeman, R. Nouicer, F. Haas, O. Dorvaux, K. Eddahbi et al., Pramana 57, 203 (2001).

[66] M. Rousseau, C. Beck, C. Bhattacharya, V. Rauch, O. Dorvaux, K. Eddahbi, C. Enaux, R. M. Freeman, F. Haas et al., Phys. Rev. C 66, 034612 (2002).

[67] I. Strojek, W. Czarnacki, W. Gawlikowicz, N. Keeley, M. Kisieliński, S. Kliczewski, A. Kordyasz, E. Koshchiy, M. Kowalczyk et al., Acta Phys. Pol. B 43, 339 (2012).

[68] E. V. Inopin and M. A. El-Moaty, Nucl. Phys. B 42, 660 (1963). 